\title{
Quantitative and Qualitative Traits of Sunflower Genotypes as Influenced by Nitrogen Application through Fertigation
}

\author{
Imran Ali Chandio ${ }^{1}$, Muhammad Nawaz Kandhro ${ }^{1 *}$, Qamaruddin Jogi ${ }^{1}$, Ghulam Murtaza Jamro ${ }^{2}$ and \\ Siraj Ahmed Channa ${ }^{3}$
}

${ }^{1}$ Department of Agronomy, Sindh Agriculture University, Tandojam, Pakistan; ${ }^{2}$ Department of Soil Science, Sindh Agriculture University, Tandojam, Pakistan; ${ }^{3}$ Department of Plant Breeding and Genetics, Sindh Agriculture University, Tandojam, Pakistan.

\begin{abstract}
Fertigation is the application of dissolved fertilizer especially nitrogen through irrigation for correcting nutrient deficiencies of plants. A field study was conducted in Tandojam for two consecutive years in autumn 2018 and 2019. The experiment was replicated thrice in randomized complete block design. Different doses of nitrogen as broadcasting and fertigation $\left(0,75,100,125 \mathrm{~kg} \mathrm{~N} \mathrm{ha}^{-1}\right.$ in two and three equal splits given at sowing time, $1^{\text {st }}, 2^{\text {nd }}$ and $3^{\text {rd }}$ irrigations, respectively) were applied to two sunflower genotypes (HO-1 and Hysun-39). Data analysis revealed nitrogen fertigation, genotypes and their interaction substantially $(\mathrm{P} \leq 0.05)$ affected growth and yield traits of sunflower. Greater seed yield $\left(\mathrm{kg} \mathrm{ha}^{-1}\right)$ and the oil content $(\%)$ were documented in $\mathrm{N}_{10}=125 \mathrm{~kg} \mathrm{~N} \mathrm{ha}^{-1}$ : three splits ( $1 / 3$ broadcasting at sowing time + fertigation- $1 / 3$ at $1^{\text {st }}$ and $1 / 3$ at $3^{\text {rd }}$ irrigation), followed by $\mathrm{N}_{8}=125 \mathrm{~kg} \mathrm{~N}^{-1}$ : two splits ( $1 / 2$ broadcasting at sowing time $+1 / 2$ as fertigation at $2^{\text {nd }}$ irrigation). In between genotypes, higher seed yield $\left(\mathrm{kg} \mathrm{ha}^{-1}\right)$ and oil content (\%) were recorded in HO-1 as compared to Hysun-39. As regards interactions, enhanced seed yield $\left(\mathrm{kg} \mathrm{ha}^{-1}\right)$ and oil content (\%) were registered in the interaction of $\mathrm{N}_{10}=125 \mathrm{~kg} \mathrm{~N} \mathrm{ha}{ }^{-1}$ : three splits $\left(1 / 3\right.$ broadcast at sowing time + fertigation- $1 / 3$ at $1^{\text {st }}$ and $1 / 3$ at $3^{\text {rd }}$ irrigation) $\times \mathrm{HO}-1$. It was concluded from the results that nitrogen dose should be divided into three splits and applied at sowing time through broadcasting and fertigated at $1^{\text {st }}$ and $2^{\text {nd }}$ irrigation, respectively. Variety HO-1 should be preferred to obtain enhanced sunflower seed yield under Tandojam climatic conditions.

Received | July 06, 2021; Accepted | November 16, 2021; Published | January 19, 2022

*Correspondence | Muhammad Nawaz Kandhro, Department of Agronomy, Sindh Agriculture University, Tandojam, Pakistan; Email: kandhromn@gmail.com

Citation | Chandio, I.A., M.N. Kandhro, Q. Jogi, G.M. Jamro and S.A. Channa.2022. Quantitative and qualitative traits of sunflower genotypes as influenced by nitrogen application through Fertigation. Pakistan Journal of Agricultural Research, 35(1): 5-14.

DOI | https://dx.doi.org/10.17582/journal.pjar/2022/35.1.5.14

Keywords | Fertigation, Genotypes, Growth, Nitrogen, Sunflower, Yield
\end{abstract}

\section{Introduction}

$\mathrm{P}$ akistan is facing acute shortage of edible oil. The demand for edible oil in Pakistan is increasing with the uncontrollable increase in population. Its indigenous production is below the consumption levels with a very wide gap between production and consumption. This gap is bridged through import of edible oil worth more than Rs. 45.0 billion annually. Pakistan ranks $3^{\text {rd }}$ largest edible oil importing country in the world (GoP, 2020). The country's oil requirement was about 2.966 million tons in which 0.83 million tons $(28 \%)$ were locally produced and the rest edible oil was imported. The edible oil requirements have increased from 0.3 million tons to 1.95 million tons. But the domestic production of edible oil has remained inadequate and fluctuating for the last couple of decades. These fluctuations are due to indigenous marketing, low support price and high cost of production which is making these crops non-profitable to March 2022 | Volume 35 | Issue 1 | Page 5 
the farmers. Only sunflower has shown some positive results in area and production compared to other oil crops (Khan and Inamullah, 2019). Oilseeds have an enormous significance as an essential part of human diet after cereal and sugar crops in Pakistan (GoP, 2019). Vegetable oil is considered as more suitable for health as compared to "ghee" from animal source because it contains less cholesterol and unsaturated fats (Masood, 2014). Sunflower is considered as the $4^{\text {th }}$ highest source of vegetable oil at world level after soybean, rapeseed and palm and $2^{\text {nd }}$ largest source of edible oil after cottonseed in Pakistan (Keerio et al., 2020). This crop fulfils about $14 \%$ cooking oils needs of Pakistan. Sunflower has the potential to bridge the gap between consumption and domestic oil production in Pakistan (Nasim et al., 2012). It is well fitted to climatic conditions and current cropping system of Pakistan (Mahmood et al., 2018). In Pakistan sunflower is being cultivated in autumn and spring (PARC, 2019). Buriro et al. (2015) has disclosed that seed of sunflower contains nearly $47 \%$ oil, linoleic acid and vitamins $\mathrm{A}, \mathrm{D}, \mathrm{E}$ and $\mathrm{K}$. The consumption of sunflower oil reduces the blood cholesterol level (Debaeke et al., 2021). Genotype selection and fertilizer application are important agro-management practices that increase crop growth and production (Sheoran et al., 2014). The hybrids are further constant and extremely self-fertile having uniformity at maturity and high yield performance (Wajid et al., 2017). Nitrogen is a plant nutrient that has direct influence on quantitative and qualitative traits of crop (Alves et al., 2017). It is the critical limiting nutrient for the plants, absorbed in plenty as nitrate, urea and ammonium. Nitrogen is a primary plant nutrient which plays an important role in plant growth and development, and ultimately causes increase or decrease in yield and quality of the crop produce (Nasim et al., 2012). The rate and source of nitrogen have a significant impact on the sunflower yield and other agronomic traits (Yassen et al., 2011). Nitrogen fertigation is the technique of applying dissolved fertilizer to crops during their growth stages through an irrigation system. It is used to spoon feeding additional nutrients or correct nutrient deficiencies detected in plant tissue analysis (Daniela et al., 2018). Nitrogen fertigation provides an excellent opportunity to maximize yield and minimize environmental pollution by increasing fertilizer use efficiency. Fertigation reduces fertilizer dose and increases return on the fertilizer (Shabbir et al., 2015). This investigation gives an insight into optimizing sunflower fertilization as well as role of nitrogen to- wards yield and yield contributing traits of sunflower. The study was aimed at evaluating the comparative impact of nitrogen application through fertigation and conventional method broadcasting on seed and oil yield of sunflower under Tandojam conditions.

\section{Materials and Methods}

The field study was conducted in Tandojam conditions during autumn 2018 and repeated in 2019. The experiment was laid out in three replicated randomized completed block design with factorial arrangements. The net plot size of experimental unit was $30 \mathrm{~m}^{2}(6 \mathrm{~m} \times 5 \mathrm{~m})$. The treatments comprised of different doses of nitrogen as broadcast and fertigation $\left(0,75,100,125 \mathrm{~kg} \mathrm{~N} \mathrm{ha}^{-1}\right.$ in two and three equal splits given at sowing time, $1^{\text {st }}, 2^{\text {nd }}$ and $3^{\text {rd }}$ irrigations, respectively) and two sunflower genotypes i.e. HO1 and Hysun-39. The land was prepared thoroughly by applying two dry ploughings, followed by heavy soaking doses. After that when soil came in workable conditions, the laser land leveler was used to maintain the level of land, followed by two cross-wise ploughings with cultivator. The bunds and feedings channel were prepared to separate each replication and treatment. The seed was sown by hand drill in separate plots during $2^{\text {nd }}$ week of August, each year. Nitrogen was applied as per treatments in the form of Urea (46\% N). Phosphorus and potassium were applied at their recommended doses of 50 and $50 \mathrm{~kg} \mathrm{ha}^{-1}$ in-the forms of SSP $\left(18 \% \mathrm{P}_{2} \mathrm{O}_{5}\right)$ and SOP $\left(50 \% \mathrm{~K}_{2} \mathrm{O}\right)$. Weeding was done before $1^{\text {st }}$ and $2^{\text {nd }}$ irrigation.

\section{Data collection}

The data was recorded on following parameters:

Plant height (cm): The plant height was measured in centimeters from base to tip with measuring tape in all tagged plants from each experimental unit and their average was calculated.

Head diameter (cm): The head diameter was measured with measuring tape in all selected plants in centimeters and the mean was computed.

Seed weight head ${ }^{-1}(\mathbf{g})$ : The weight of seeds head ${ }^{-1}$ was measured by using digital weight balance and the average was calculated.

Biological yield ( $\left.\mathrm{kg} \mathrm{ha}^{-1}\right)$ : The biomass of total harvested and dried plants was measured in kilograms 

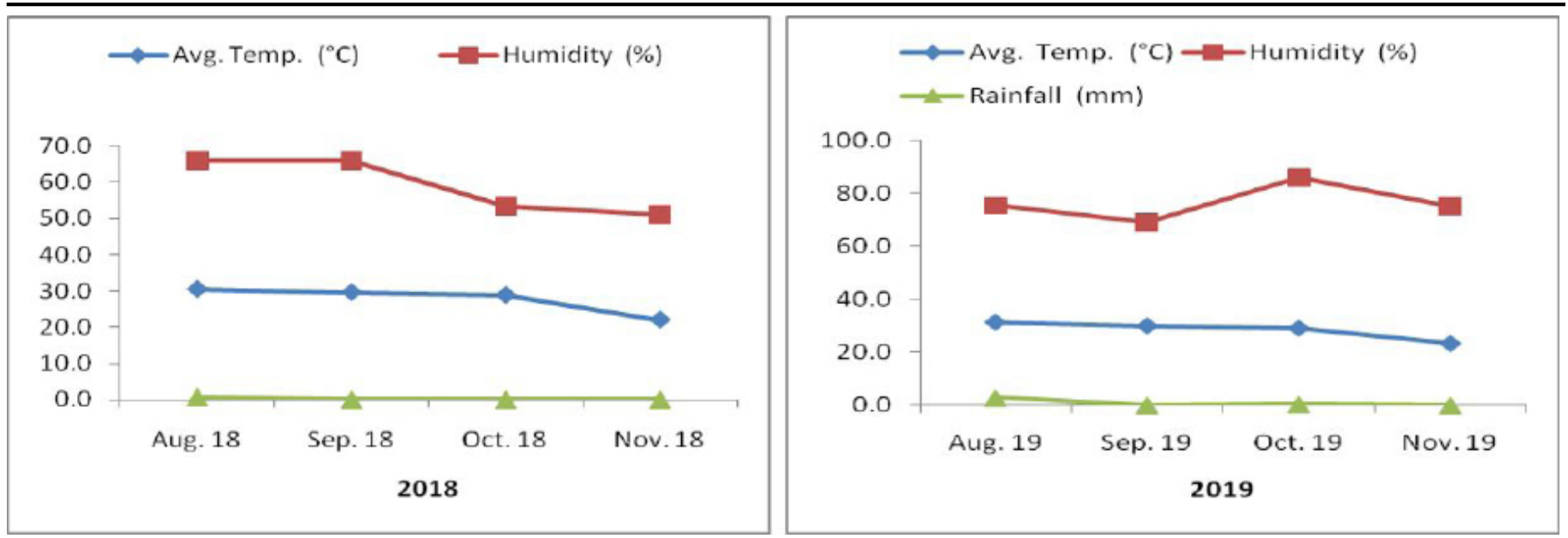

Figure 1: Tandojam weather data of 2018 and 2019 during sunflower growth period.

Table 1: Physico-chemical analysis of experimental soil (2018 and 2019).

\section{Soil Parameter}

Soil texture

Clay (\%)

Silt (\%)

41.50

Sand (\%)

Textural class

Chemical properties

Soil $\mathrm{pH}$

$\mathrm{EC}\left(\mathrm{dS} \mathrm{m}^{-1}\right)$

2.370

Organic matter (\%)

0.500

$\mathrm{P}$ available $\left(\mathrm{mg} \mathrm{kg}^{-1}\right)$

9.000

$\mathrm{K}$ extractable $\left(\mathrm{mg} \mathrm{kg}^{-1}\right)$

0.890

Total N (\%)

by using digital weight balance before threshing seeds from heads and then average was calculated.

Seed yield ( $\left.\mathbf{k g ~ h a} \mathbf{~ h}^{-1}\right)$ : The seed obtained from heads of total harvested plants after drying was weighed in kilograms by using digital weight balance average was worked out.

Oils content (\%): The oil content was determined by taking 10 grams of seed through Soxhlete apparatus (for each treatment) available at Institute of Oilseeds Research, ARC, Tandojam. The oil content \% was measured by using the formula: Oil weight $(\mathrm{g}) \div$ Seed sample weight $(\mathrm{g}) \times 100$.

Chlorophyll content ( $\left.\mathrm{mg} \mathrm{g}^{-1}\right)$ : The chlorophyll content was recorded in $\mathrm{mg} \mathrm{g}^{-1}$ with digital chlorophyll meter from the leaves of living plants in the field during peak vegetative growth of the plants.
Crop growth rate $\left(\mathrm{g} \mathrm{m}^{-2} \mathrm{day}^{-1}\right)$ : The growth rate of crop was noted from the plants selected at random through the formula: (Weight $2-$ Weight 1 ) $\div$ (Time 2 -Time 1) at the climax of growth period of crop.

\section{Statistical analysis}

The recorded data was analyzed statistically through the application of analysis of variance technique by involving software Statistix version 8.1 (Statistix, 2006). The LSD test was applied 0.05 for evaluating the difference of various treatments.

\section{Physico-chemical analysis of soil}

The Soil Auger was used for taking soil samples before sowing and after harvesting of crop from different five locations of the experimental area at the depth of 0-30 $\mathrm{cm}$. The samples were dried under sun, then grinding and sieving in $2 \mathrm{~mm}$ was done and later on kept in containers of plastic. After that different properties of physical as well as chemical nature of the soil were done. The procedure adopted was that of Rayan et al. (2001) (Table 1).

\section{Weather data}

Tandojam weather data during growing season of crop was collected from the Meteorological Station established at Tandojam. The weather data on monthly basis is given as under in the Figure 1.

\section{Results and Discussion}

\section{Plant height (cm)}

Nitrogen application through fertigation caused marked $(\mathrm{P}<0.05)$ effects on plant height $(\mathrm{cm})$ of sunflower genotypes (Table 2). Tallest plants were observed under $\mathrm{N}_{10}=125 \mathrm{~kg} \mathrm{~N} \mathrm{ha}^{-1}$ : three splits $(1 / 3$ broadcasting at sowing time + fertigation $-1 / 3$ at $1^{\text {st }}$ and $1 / 3$ 
Table 2: Plant height $(\mathrm{cm})$ and head diameter $(\mathrm{cm})$ of sunflower as affected by nitrogen fertigation and genotypes.

\section{Fertigation method}

Plant height (cm)

Genotypes Mean

HO-1 Hysun-39

$\mathrm{N}_{1}$ Control (No nitrogen)

$\mathrm{N}_{2} 75 \mathrm{~kg} \mathrm{~N} \mathrm{ha}^{-1}: 2$ splits ( $1 / 2$ broadcasting at sowing time $+1 / 2$ as fertigation at $2^{\text {nd }}$ irrigation)

$\mathrm{N}_{3} 75 \mathrm{~kg} \mathrm{~N} \mathrm{ha}^{-1}: 2$ splits $\left(1 / 2\right.$ at $1^{\text {st }}$ irrigation $+1 / 2$ at $3^{\text {rd }}$; both as fertigation)

$\mathrm{N}_{4} 75 \mathrm{~kg} \mathrm{~N} \mathrm{ha} \mathrm{k}^{-1}: 3$ splits ( $1 / 3$ broadcasting at sowing time; + fertigation- $1 / 3$ at $1^{\text {st }}$ irrigation and $1 / 3$ at $3^{\text {rd }}$ irrigation)

$\mathrm{N}_{5} 100 \mathrm{~kg} \mathrm{~N} \mathrm{ha}{ }^{-1}: 2$ splits ( $1 / 2$ broadcasting at sowing time $+1 / 2$ as fertigation at $2^{\text {nd }}$ irrigation)

$\mathrm{N}_{6} 100 \mathrm{~kg} \mathrm{~N} \mathrm{ha}{ }^{-1}: 2$ splits ( $1 / 2$ at $1^{\text {st }}$ irrigation $+1 / 2$ at $3^{\text {rd }}$ irrigation; $204.0 \quad 203.7$ both as fertigation)

$\mathrm{N}_{7} 100 \mathrm{~kg} \mathrm{~N} \mathrm{ha}{ }^{-1}: 3$ splits ( $1 / 3$ broadcasting at sowing time; + ferti- 206.0 gation $-1 / 3$ at $1^{\text {st }}$ irrigation and $1 / 3$ at $3^{\text {rd }}$ irrigation)

$\mathrm{N}_{8} 125 \mathrm{~kg} \mathrm{~N} \mathrm{ha}^{-1}: 2$ splits ( $1 / 2$ broadcasting at sowing time $+1 / 2$ as 228.3 fertigation at $2^{\text {nd }}$ irrigation)

$\mathrm{N}_{9} 125 \mathrm{~kg} \mathrm{~N} \mathrm{ha}{ }^{-1}: 2$ splits ( $1 / 2$ at $1^{\text {st }}$ irrigation $+1 / 2$ at $3^{\text {rd }}$ irrigation; 220.3 both as fertigation)

$\mathrm{N}_{10} 125 \mathrm{~kg} \mathrm{~N} \mathrm{ha}{ }^{-1}: 3$ splits (1/3 broadcasting at sowing time; + ferti- 231.7227 .0 gation $-1 / 3$ at $1^{\text {st }}$ irrigation and $1 / 3$ at $3^{\text {rd }}$ irrigation)

\section{Mean}

Variable

CV

SE

$P$ value

$\mathrm{LSD}_{0.05}$

$\begin{array}{llllll}204.3 & 200.1 & -- & 35.0 \text { A } & 32.4 \text { B } & -- \\ \text { Fert (F) } & \text { Geno }(G) & \text { Fx G } & \text { Fert (F) } & \text { Geno }(G) & \text { Fx G } \\ 11.53 & & & 12.61 & & \\ 13.459 & 6.0191 & 19.034 & 2.453 & 1.097 & 3.469 \\ 0.005 & 0.489 & 1.000 & 0.000 & 0.027 & 1.000 \\ 27.246 & - & - & 4.967 & 2.221 & -\end{array}$

Head diameter $(\mathrm{cm})$

Genotypes Mean

HO-1 Hysun-39

$27.5 \quad 26.8$

$27.3 \mathrm{D}$

$28.1 \mathrm{D}$

184.2 DE 29.3 27.0

$\begin{array}{llll}\begin{array}{l}191.5 \\ \text { CDE }\end{array} & 32.8 & 30.3 & 31.6 \mathrm{CD} \\ \text { 193.3 DE } & 33.5 & 30.8 & 32.2 \mathrm{BCD}\end{array}$

$\begin{array}{llll}198.2 & 35.7 & 32.5 & 34.1 \mathrm{BC}\end{array}$

BCD

$\begin{array}{llll}203.8 & 36.0 & 33.7 & 34.8 \mathrm{BC}\end{array}$

A-D

$206.5 \quad 37.2 \quad 34.7 \quad 35.9 \mathrm{ABC}$

A-D

$\begin{array}{llll}222.8 \mathrm{AB} & 38.2 & 35.7 & 36.9 \mathrm{AB}\end{array}$

$\begin{array}{llll}216.5 & 37.7 & 34.8 & 36.3 \mathrm{ABC}\end{array}$

229.3 A $\quad 41.7 \quad 38.0 \quad 39.8$ A at $3^{\text {rd }}$ irrigation), followed by $\mathrm{N}_{8}=125 \mathrm{~kg} \mathrm{~N} \mathrm{ha}^{-1}$ : two splits $(1 / 2$ broadcasting at sowing time $+1 / 2$ as fertigation at $2^{\text {nd }}$ irrigation). However, smallest plants were noted under $\mathrm{N}_{1}=$ control (no nitrogen). Among genotypes, taller plants were noticed in HO-1 whereas smaller ones were registered in Hysun-39. In case of interactive effects, greatest height of plants was obtained in the interaction of $\mathrm{N}_{10}=125 \mathrm{~kg} \mathrm{~N} \mathrm{ha}^{-1}$ : three splits $(1 / 3$ broadcasting at sowing time + fertigation $-1 / 3$ at $1^{\text {st }}$ and $1 / 3$ at $3^{\text {rd }}$ irrigation) with genotype $\mathrm{HO}-1$ while lowest plant height was registered in the interaction of $\mathrm{N}_{1}=$ control (no nitrogen) with genotype Hysun-39.

\section{Head diameter $(\mathrm{cm})$}

The analysis of data indicated that application of nitrogen through fertigation substantially $(\mathrm{P}<0.05)$ influenced on head diameter $(\mathrm{cm})$ of sunflower $(\mathrm{Ta}-$ ble 2). The largest head diameter was recorded under $\mathrm{N}_{10}=125 \mathrm{~kg} \mathrm{~N} \mathrm{ha}^{-1}$ : three splits (1/3 broadcasting at sowing time + fertigation- $1 / 3$ at $1^{\text {st }}$ and $1 / 3$ at $3^{\text {rd }}$ irri- gation). Whereas, $\mathrm{N}_{8}=125 \mathrm{~kg} \mathrm{~N} \mathrm{ha}{ }^{-1}$ : two splits ( $1 / 2$ broadcasting at sowing time $+1 / 2$ as fertigation at $2^{\text {nd }}$ irrigation) ranked $2^{\text {nd }}$ in head diameter. Nevertheless, lowest head diameter was documented under $\mathrm{N}_{1}=$ control (no nitrogen). Genotype $\mathrm{HO}-1$ gave greater head diameter over Hysun-39. The interaction of $\mathrm{N}_{10}=125 \mathrm{~kg} \mathrm{~N} \mathrm{ha}^{-1}$ : three splits $(1 / 3$ broadcasting at sowing time + fertigation $-1 / 3$ at $1^{\text {st }}$ and $1 / 3$ at $3^{\text {rd }}$ irrigation) with genotype HO-1 produced greatest head diameter and that of $\mathrm{N}_{1}=$ control (no nitrogen) with Hysun-39 gave least head diameter $(\mathrm{cm})$.

\section{Seed weight head ${ }^{-1}(g)$}

The data analysis revealed that seed weight head ${ }^{-1}(\mathrm{~g})$ of sunflower was affected markedly $(\mathrm{P} \leq 0.05)$ by application of nitrogen through fertigation (Table 3 ). In case of nitrogen application methods, $\mathrm{N}_{10}=125 \mathrm{~kg} \mathrm{~N}$ $\mathrm{ha}^{-1}$ : three splits $(1 / 3$ broadcasting at sowing time + fertigation- $1 / 3$ at $1^{\text {st }}$ and $1 / 3$ at $3^{\text {rd }}$ irrigation) gave higher seed weight head ${ }^{-1}(\mathrm{~g}) . \mathrm{N}_{8}=125 \mathrm{~kg} \mathrm{~N} \mathrm{ha}^{-1}$ : two splits $\left(1 / 2\right.$ broadcasting at sowing time $+1 / 2$ as fertigation at $2^{\text {nd }}$ 
Table 3: Seed weight head ${ }^{-1}(\mathrm{~g})$ and biological yield $\left(\mathrm{kg} \mathrm{ha}^{-1}\right)$ of ssunflower as affected by nitrogen fertigation and genotypes.

\section{Fertigation method}

$\mathrm{N}_{1}$ Control (No nitrogen)

$\mathrm{N}_{2} 75 \mathrm{~kg} \mathrm{~N} \mathrm{ha}{ }^{-1}: 2$ splits ( $1 / 2$ fertigation at $2^{\text {nd }}$ irrigation)

$\mathrm{N}_{3} 75 \mathrm{~kg} \mathrm{~N} \mathrm{ha}^{-1}: 2$ splits ( $1 / 2$ at $1^{\text {st }}$ irrigation $+1 / 2$ at $3^{\text {rd }}$; both as fertigation)

$\mathrm{N}_{4} 75 \mathrm{~kg} \mathrm{~N} \mathrm{ha}{ }^{-1}: 3$ splits ( $1 / 3$ broadcasting at sowing time; + fertigation- $1 / 3$ at $1^{\text {st }}$ irrigation and $1 / 3$ at $3^{\text {rd }}$ irrigation)

$\mathrm{N}_{5} 100 \mathrm{~kg} \mathrm{~N} \mathrm{ha} a^{-1}: 2$ splits (1/2 broadcasting at sowing time $+1 / 2$ as fertigation at $2^{\text {nd }}$ irrigation)

$\mathrm{N}_{6} 100 \mathrm{~kg} \mathrm{~N} \mathrm{ha} \mathrm{k}^{-1}: 2$ splits ( $1 / 2$ at $1^{\text {st }}$ irrigation $+1 / 2$ at $3^{\text {rd }}$ irrigation; both as fertigation)

$\mathrm{N}_{7} 100 \mathrm{~kg} \mathrm{~N} \mathrm{ha}{ }^{-1}: 3$ splits ( $1 / 3$ broadcasting at sowing time; + fertigation- $1 / 3$ at $1^{\text {st }}$ irrigation and $1 / 3$ at $3^{\text {rd }}$ irrigation)

$\mathrm{N}_{8} 125 \mathrm{~kg} \mathrm{~N} \mathrm{ha}^{-1}: 2$ splits ( $1 / 2$ broadcasting at sowing time $+1 / 2$ as fertigation at $2^{\text {nd }}$ irrigation)

$\mathrm{N}_{9} 125 \mathrm{~kg} \mathrm{~N} \mathrm{ha}{ }^{-1}: 2$ splits ( $1 / 2$ at $1^{\text {st }}$ irrigation $+1 / 2$ at $3^{\text {rd }}$ irrigation; both as fertigation)

$\mathrm{N}_{10} 125 \mathrm{~kg} \mathrm{~N} \mathrm{ha}^{-1}: 3$ splits ( $1 / 3$ broadcasting at sowing time; + fertigation- $1 / 3$ at $1^{\text {st }}$ irrigation and $1 / 3$ at $3^{\text {rd }}$ irrigation)

Mean

Variable

CV

SE

$P$ value

$\mathrm{LSD}_{0.05}$

irrigation) trailed in efficacy of seed weight head $^{-1}$. However, minimal seed weight head ${ }^{-1}$ was noted in $\mathrm{N}_{1}=$ control (no nitrogen). As much as genotypes are concerned, the highest seed weight head ${ }^{-1}$ was observed in HO-1 whereas lowest seed weight head ${ }^{-1}$ was registered in Hysun-39. With regard to interactive effects, greatest seed weight head ${ }^{-1}$ was registered in $\mathrm{N}_{10}=125 \mathrm{~kg} \mathrm{~N} \mathrm{ha}^{-1}$ : three splits $(1 / 3$ broadcasting at sowing time + fertigation- $1 / 3$ at $1^{\text {st }}$ and $1 / 3$ at $3^{\text {rd }}$ irrigation) with genotype $\mathrm{HO}-1$ while least seed weight head $^{-1}(\mathrm{~g})$ was observed when $\mathrm{N}_{1}=$ control (no nitrogen) was interacted with Hysun-39.

\section{Biological yield $\left(\mathrm{kg} \mathrm{ha}^{-1}\right)$}

Nitrogen application through fertigation demonstrated considerable $(\mathrm{P} \leq 0.05)$ effects over the biological yield of sunflower (Table 3). Enhanced biological yield $\left(\mathrm{kg} \mathrm{ha}^{-1}\right)$ was recorded under $\mathrm{N}_{10}=125 \mathrm{~kg} \mathrm{~N}$ ha ${ }^{1}$ : three splits $(1 / 3$ broadcasting at sowing time + fertigation $-1 / 3$ at $1^{\text {st }}$ and $1 / 3$ at $3^{\text {rd }}$ irrigation), followed by $\mathrm{N}_{8}$

\begin{tabular}{|c|c|c|c|c|c|}
\hline \multicolumn{3}{|c|}{ Seed weight head ${ }^{-1}(\mathrm{~g})$} & \multicolumn{3}{|c|}{ Biological yield (kg ha $\left.{ }^{-1}\right)$} \\
\hline \multicolumn{2}{|c|}{ Genotypes } & \multirow{2}{*}{ Mean } & \multicolumn{2}{|c|}{ Genotypes } & \multirow[t]{2}{*}{ Mean } \\
\hline HO-1 & Hysun-39 & & HO-1 & Hysun-39 & \\
\hline 177.7 & 378.0 & $440.5 \mathrm{C}$ & 3624 & 3520 & $3572 \mathrm{C}$ \\
\hline 188.0 & 387.3 & $452.5 \mathrm{BC}$ & 3986 & 3656 & $3821 \mathrm{BC}$ \\
\hline 193.0 & 415.3 & $466.8 \mathrm{BC}$ & 4078 & 4040 & $4059 \mathrm{AB}$ \\
\hline 194.7 & 425.0 & $485.3 \mathrm{BC}$ & 4114 & 4045 & $4080 \mathrm{AB}$ \\
\hline 199.7 & 441.7 & $494.8 \mathrm{BC}$ & 4119 & 4117 & $4118 \mathrm{AB}$ \\
\hline 204.0 & 442.3 & 497.0 BC & 4173 & 4158 & $4165 \mathrm{AB}$ \\
\hline 206.0 & 482.7 & $520.5 \mathrm{ABC}$ & 4211 & 4163 & $4187 \mathrm{AB}$ \\
\hline 220.3 & 493.3 & $532.7 \mathrm{AB}$ & 4353 & 4250 & $4302 \mathrm{~A}$ \\
\hline 228.3 & 488.0 & $523.3 \mathrm{AB}$ & 4253 & 4246 & $4249 \mathrm{AB}$ \\
\hline 231.7 & 530.3 & $584.5 \mathrm{~A}$ & 4379 & 4287 & $4333 \mathrm{~A}$ \\
\hline $551.2 \mathrm{~A}$ & $448.4 \mathrm{~B}$ & -- & 4129 & 4048 & -- \\
\hline Fert $(\mathrm{F})$ & Geno $(\mathrm{G})$ & $F \times G$ & Fert $(F)$ & Geno $(\mathrm{G})$ & $F \times G$ \\
\hline 13.87 & & & 9.24 & & \\
\hline 40.021 & 17.898 & 56.599 & 218.21 & 97.586 & 308.59 \\
\hline 0.038 & 0.000 & 0.998 & 0.037 & 0.414 & 0.999 \\
\hline 81.019 & 36.233 & - & 441.74 & - & - \\
\hline
\end{tabular}

$=125 \mathrm{~kg} \mathrm{~N} \mathrm{ha}^{-1}$ : two splits $(1 / 2$ broadcasting at sowing time $+1 / 2$ as fertigation at $2^{\text {nd }}$ irrigation). Nonetheless, lowest biological yield $\left(\mathrm{kg} \mathrm{ha}^{-1}\right)$ was noted under $\mathrm{N}_{1}$ = control (no nitrogen). Genotype HO-1 surpassed Hysun-39 in biological yield $\left(\mathrm{kg} \mathrm{ha}^{-1}\right)$. As regards interaction, greatest biological yield $\left(\mathrm{kg} \mathrm{ha}^{-1}\right)$ was obtained in $\mathrm{N}_{10}=125 \mathrm{~kg} \mathrm{~N}^{-1}$ : three splits (1/3 broadcasting at sowing time + fertigation $-1 / 3$ at $1^{\text {st }}$ and $1 / 3$ at $3^{\text {rd }}$ irrigation) $\times$ HO-1 whereas minimum biological yield $\left(\mathrm{kg} \mathrm{ha}^{-1}\right)$ noticed in $\mathrm{N}_{1}=$ control (no nitrogen) x Hysun-39.

\section{Seed yield $\left(k g h a^{-1}\right)$}

The statistical analysis envisaged that nitrogen application through fertigation demonstrated marked $(\mathrm{P} \leq 0.05)$ impact on the seeds yield (Table 4). Better seed yield $\left(\mathrm{kg} \mathrm{ha}^{-1}\right)$ was documented in $\mathrm{N}_{10}=125 \mathrm{~kg}$ $\mathrm{N} \mathrm{ha}^{-1}$ : three splits $(1 / 3$ broadcasting at sowing time + fertigation $-1 / 3$ at $1^{\text {st }}$ and $1 / 3$ at $3^{\text {rd }}$ irrigation). $N_{8}=$ $125 \mathrm{~kg} \mathrm{~N} \mathrm{~N}^{-1}$ : two splits $(1 / 2$ broadcasting at sowing 
Table 4: Seed yield ( $\left.\mathrm{kg} \mathrm{ha}^{-1}\right)$ and oil content (\%) of sunflower as affected by nitrogen fertigation and genotypes.

\section{Fertigation method}

\section{Seed yield $\left(\mathrm{kg} \mathrm{ha}^{-1}\right)$}

Genotypes

HO-1 Hysun-39

$177.7 \quad 2128$

$188.0 \quad 2270$

$193.0 \quad 2331$

194.72376

$199.7 \quad 2431$

$\mathrm{N}$ gation at $2^{\text {nd }}$ irrigation)

$\mathrm{N}_{6} 100 \mathrm{~kg} \mathrm{~N} \mathrm{ha}^{-1}: 2$ splits (1/2 at $1^{\text {st }}$ irrigation $+1 / 2$ at $3^{\text {rd }}$ irrigation; both 204.02435 as fertigation)

$\mathrm{N}_{7} 100 \mathrm{~kg} \mathrm{~N} \mathrm{ha}^{-1}: 3$ splits (1/3 broadcasting at sowing time; + fertiga- $\quad 206.02482$ tion $-1 / 3$ at $1^{\text {st }}$ irrigation and $1 / 3$ at $3^{\text {rd }}$ irrigation)

$\mathrm{N}_{8} 125 \mathrm{~kg} \mathrm{~N} \mathrm{ha}^{-1}: 2$ splits (1/2 broadcasting at sowing time + $1 / 2$ as ferti- 220.32535 gation at $2^{\text {nd }}$ irrigation)

$\mathrm{N}_{9} 125 \mathrm{~kg} \mathrm{~N} \mathrm{ha}^{-1}: 2$ splits $\left(1 / 2\right.$ at $1^{\text {st }}$ irrigation $+1 / 2$ at $3^{\text {rd }}$ irrigation; both as fertigation)

$\mathrm{N}_{10} 125 \mathrm{~kg} \mathrm{~N} \mathrm{ha}^{-1}: 3$ splits (1/3 broadcasting at sowing time; + fertigation $-1 / 3$ at $1^{\text {st }}$ irrigation and $1 / 3$ at $3^{\text {rd }}$ irrigation)

\section{Mean}

Variable

$\mathrm{CV}$

$\mathrm{SE}$

$P$ value

$\mathrm{LSD}_{0.05}$

time $+1 / 2$ as fertigation at $2^{\text {nd }}$ irrigation) ranked $2^{\text {nd }}$ in performance. Least seed yield was observed in $\mathrm{N}_{1}$ = control (o nitrogen). Among genotypes, more seed yield $\left(\mathrm{kg} \mathrm{ha}^{-1}\right)$ was observed in HO-1 as compared to Hysun-39. The interaction of $\mathrm{N}_{10}=125 \mathrm{~kg} \mathrm{~N} \mathrm{ha}{ }^{-1}$ : three splits $(1 / 3$ broadcasting at sowing time + fertigation- $1 / 3$ at $1^{\text {st }}$ and $1 / 3$ at $3^{\text {rd }}$ irrigation) with genotype HO-1 was found best and produced improved seed yield $\left(\mathrm{kg} \mathrm{ha}^{-1}\right)$ in contrast to other interactions. Nonetheless, the interaction of $\mathrm{N}_{1}=$ control (no nitrogen) with genotype Hysun-39 resulted in lowest seed yield $\left(\mathrm{kg} \mathrm{ha}{ }^{-1}\right)$.

\section{Oil content (\%)}

Nitrogen application through fertigation caused significant $(\mathrm{P}>0.05)$ effect on oil content $(\%)$ of sunflower (Table 4). In case of nitrogen application methods numerically greater oil content was recorded in $\mathrm{N}_{10}=$ $125 \mathrm{~kg} \mathrm{~N} \mathrm{ha}^{-1}$ : three splits ( $1 / 3$ broadcasting at sowing time + fertigation $-1 / 3$ at $1^{\text {st }}$ and $1 / 3$ at $3^{\text {rd }}$ irrigation), followed by $\mathrm{N}_{8}=125 \mathrm{~kg} \mathrm{~N}^{-1}$ : two splits ( $1 / 2$ broad- casting at sowing time $+1 / 2$ as fertigation at $2^{\text {nd }}$ irrigation). Nevertheless, least oil content (\%) was observed in $\mathrm{N}_{1}=$ control (no nitrogen). As regards genotypes, superior oil content (\%) was observed in $\mathrm{HO}-1$ over genotype Hysun-39. In case of interactive effects, numerically more oil content (\%) was noticed in $\mathrm{N}_{10}=$ $125 \mathrm{~kg} \mathrm{~N} \mathrm{ha}{ }^{-1}$ : three splits ( $1 / 3$ broadcasting at sowing time + fertigation- $1 / 3$ at $1^{\text {st }}$ and $1 / 3$ at $3^{\text {rd }}$ irrigation) $x$ HO-1 while minimal oil content was observed in the $\mathrm{N}_{1}=$ control (no nitrogen) interacted with Hysun-39.

\section{Chlorophyll content ( $\mu$ mol $m^{-2}$ of leaf)}

The effect of nitrogen application by fertigation methods was not substantial $(\mathrm{P}>0.05)$ on chlorophyll content ( $\mu \mathrm{mol} \mathrm{m} \mathrm{m}^{-2}$ of leaf) of sunflower (Table 5). As regards nitrogen application methods, numerically

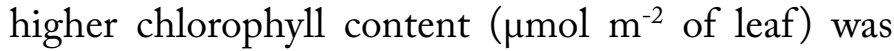
noted under $\mathrm{N}_{10}=125 \mathrm{~kg} \mathrm{~N}^{-1}$ : three splits $(1 / 3$ broadcasting at sowing time + fertigation- $1 / 3$ at $1^{\text {st }}$ and $1 / 3$ at $3^{\text {rd }}$ irrigation) whereas, $\mathrm{N}_{8}=125 \mathrm{~kg} \mathrm{~N} \mathrm{ha}^{-1}$ : two splits $(1 / 2$ broadcasting at sowing time $+1 / 2$ as fertigation 
Table 5: Chlorophyll content ( $\mu$ mol $m^{-2}$ of leaf) and crop growth rate $\left(\mathrm{g} \mathrm{m}^{-2}\right.$ day $\left.y^{-1}\right)$ of sunflower as affected by nitrogen fertigation and genotypes.

\section{Fertigation method}

$\mathrm{N}_{1}$ Control (No nitrogen)

$\mathrm{N}_{2} \quad 75 \mathrm{~kg} \mathrm{~N} \mathrm{ha}{ }^{-1}: 2$ splits ( $1 / 2$ broadcasting at sowing time $+1 / 2$ as fertigation at $2^{\text {nd }}$ irrigation)

$\mathrm{N}_{3} \quad 75 \mathrm{~kg} \mathrm{~N} \mathrm{ha}{ }^{-1}: 2$ splits $\left(1 / 2\right.$ at $1^{\text {st }}$ irrigation $+1 / 2$ at $3^{\text {rd }}$; both as fertigation)

$\mathrm{N}_{4} \quad 75 \mathrm{~kg} \mathrm{~N} \mathrm{ha} \mathrm{N}^{-1}: 3$ splits ( $1 / 3$ broadcasting at sowing time; + fertigation- $1 / 3$ at $1^{\text {st }}$ irrigation and $1 / 3$ at $3^{\text {rd }}$ irrigation)

$\mathrm{N}_{5} \quad 100 \mathrm{~kg} \mathrm{~N} \mathrm{ha}{ }^{-1}: 2$ splits ( $1 / 2$ broadcasting at sowing time $+1 / 2$ as fertigation at $2^{\text {nd }}$ irrigation)

$\mathrm{N}_{6} 100 \mathrm{~kg} \mathrm{~N} \mathrm{ha}{ }^{-1}: 2$ splits $\left(1 / 2\right.$ at $1^{\text {st }}$ irrigation $+1 / 2$ at $3^{\text {rd }}$ irrigation; both 204.045 .7 as fertigation)

$\mathrm{N}_{7} \quad 100 \mathrm{~kg} \mathrm{~N} \mathrm{ha}{ }^{-1}: 3$ splits ( $1 / 3$ broadcasting at sowing time; + fertigation- $1 / 3$ at $1^{\text {st }}$ irrigation and $1 / 3$ at $3^{\text {rd }}$ irrigation)

$\mathrm{N}_{8} \quad 125 \mathrm{~kg} \mathrm{~N} \mathrm{ha} \mathrm{N}^{-1}: 2$ splits ( $1 / 2$ broadcasting at sowing time $+1 / 2$ as fertigation at $2^{\text {nd }}$ irrigation)

$\mathrm{N}_{9} \quad 125 \mathrm{~kg} \mathrm{~N} \mathrm{ha}^{-1}: 2$ splits ( $1 / 2$ at $1^{\text {st }}$ irrigation $+1 / 2$ at $3^{\text {rd }}$ irrigation; both 228.349 .7 as fertigation)

$\mathrm{N}_{10} \quad 125 \mathrm{~kg} \mathrm{~N} \mathrm{ha}{ }^{-1}: 3$ splits ( $1 / 3$ broadcasting at sowing time; + fertiga- $231.7 \quad 53.0$ tion- $1 / 3$ at $1^{\text {st }}$ irrigation and $1 / 3$ at $3^{\text {rd }}$ irrigation)

\section{Mean}

Variable

CV

SE

$P$ value

$\mathrm{LSD}_{0.05}$

$206.0 \quad 47.0$

Chlorophyll content ( $\mu \mathrm{mol}$ Crop growth rate $\left(\mathrm{g} \mathrm{m}^{-2}\right.$

$\mathbf{m}^{-2}$ )

Genotypes

HO-1 Hysun-39

$177.7 \quad 43.2$

$188.0 \quad 43.5$

$193.0 \quad 44.2$

$194.7 \quad 44.3$

$199.7 \quad 44.4$

$220.3 \quad 52.5$

49.7

$48.3 \quad 46.9$

Fert (F) Geno (G)

13.11

$3.600 \quad 1.610$

$0.061 \quad 0.370$
Mean Genotypes

Mean

HO-1 Hysun-39

$\begin{array}{llll}43.2 & 1.5 & 1.4 & 1.4 \mathrm{C}\end{array}$

$44.1 \quad 1.9 \quad 1.4$

$1.4 \mathrm{BC}$

$\begin{array}{lll}44.5 & 2.2 & 1.8\end{array}$

2.0 BC

$\begin{array}{llll}45.3 & 2.3 & 1.9 & 2.1 \mathrm{BC}\end{array}$

$2.2 \mathrm{BC}$

$48.9 \quad 2.6 \quad 2.5$

$2.5 \mathrm{~B}$

$52.9 \quad 2.9$

$\begin{array}{lll}50.7 & 2.7 \quad 2.6\end{array}$

$2.6 \mathrm{AB}$

$53.2 \quad 3.4 \quad 2.8$

$3.1 \mathrm{~A}$

$-$

$--\quad 2.6$

$2.6 \quad 2.1$

Fx G Fert 39.67

$\begin{array}{lll}5.092 & 0.538 & 0.240\end{array}$

0.761

$\begin{array}{lll}1.000 & 0.018 & 0.098\end{array}$

0.972 at $2^{\text {nd }}$ irrigation) ranked $2^{\text {nd }}$. However, minimum chlorophyll content ( $\mu \mathrm{mol} \mathrm{m} \mathrm{m}^{-2}$ of leaf) was noted un$\operatorname{der} \mathrm{N}_{1}=$ control (no nitrogen). Genotype HO-1 gave

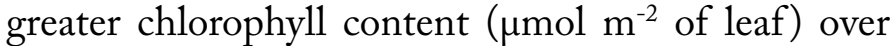
Hysun-39. The interaction of $\mathrm{N}_{10}=125 \mathrm{~kg} \mathrm{~N} \mathrm{ha}^{-1}$ : three splits $(1 / 3$ broadcasting at sowing time + fertigation- $1 / 3$ at $1^{\text {st }}$ and $1 / 3$ at $3^{\text {rd }}$ irrigation) with genotype $\mathrm{HO}-1$ resulted in better chlorophyll content $(\mu \mathrm{mol}$ $\mathrm{m}^{-2}$ of leaf).

\section{Crop growth rate $\left(\mathrm{g} \mathrm{m}^{-2}\right.$ day $\left.\mathrm{y}^{-1}\right)$}

Nitrogen application through fertigation exhibited substantial $(\mathrm{P}<0.05)$ effects on crop growth rate $\left(\mathrm{g} \mathrm{m}^{-2}\right.$ day $^{-1}$ ) of sunflower (Table 5). As regards $\mathrm{N}$ application methods higher growth rate of crop was noticed in $\mathrm{N}_{10}=125 \mathrm{~kg} \mathrm{~N} \mathrm{ha}^{-1}$ : three splits $(1 / 3$ broadcasting at sowing time + fertigation $-1 / 3$ at $1^{\text {st }}$ and $1 / 3$ at $3^{\text {rd }}$ irri-

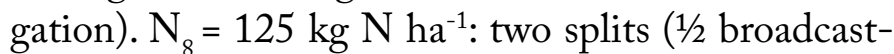
ing at sowing time $+1 / 2$ as fertigation at $2^{\text {nd }}$ irrigation) proved $2^{\text {nd }}$ in efficacy of giving crop growth rate $(\mathrm{g}$ $\mathrm{m}^{-2}$ day $\left.^{-1}\right)$. However, minimal growth rate of sunflower crop was noted in $\mathrm{N}_{1}=$ control (no nitrogen). As much as genotypes are concerned, numerically higher growth rate was observed in $\mathrm{HO}-1$ whereas lowest one was registered in Hysun-39. In case of interactive effects, numerically better growth rate $\left(\mathrm{g} \mathrm{m}^{-2}\right.$ day $\left.^{-1}\right)$ was documented in $\mathrm{N}_{10}=125 \mathrm{~kg} \mathrm{~N} \mathrm{ha}^{-1}$ : three splits $\left(1 / 3\right.$ broadcasting at sowing time + fertigation- $1 / 3$ at $1^{\text {st }}$ and $1 / 3$ at $3^{\text {rd }}$ irrigation) with genotype $\mathrm{HO}-1$.

Nitrogen is an essential plant nutrient to stimulate plant growth and development and ultimately yield and quality of the produce (U1lah et al., 2015). Nitrogen application at optimum rate improves the soil nitrogen status and subsequently causes increased crop yields (Kandil et al., 2017). Among the modern agro-management practices fertilizer application are imperative for boosting the growth and production March 2022 | Volume 35 | Issue 1 | Page 11 
of sunflower. The farmers in our country generally use the old method of fertilization application i.e. broadcasting. This method has so many disadvantages like uneven distributions of seeds, depth, and seed lying scattered being picked up by birds (Amin et al., 2006). Furthermore, nitrogen use efficiency is very low (3040\%) (Kumar et al., 2021). The findings of this study advocated that performance of sunflower almost in all growth and yield parameters was better when nitrogen was applied @ $125 \mathrm{~kg} \mathrm{~N} \mathrm{ha}{ }^{-1}$ : three splits $(1 / 3$ broadcasting at sowing time + fertigation $-1 / 3$ at $1^{\text {st }}$ and $1 / 3$ at $3^{\text {rd }}$ irrigation). This superior performance may be attributed to most favorable dose and time of nitrogen application which rewarded the crop nutrient requirements at proper stage. Crops nutrient requirements vary at different growth stages. Nitrogen is the most important plant nutrient which is needed by plants in larger quantity and at different stages. When required nutrient is supplied to crop at proper time and in required quantity, the positive results could be achieved. The nutrients must be made available to the plants at the proper growth stage so as to avoid their excessive loss. Through fertigation fertilizers are applied in soluble form at critical growth stages with higher demand for nutrients. It ensures a regular and timely supply of nutrients without contaminating the environment through leaching (Asad et al., 2002). Fertigation is the technique of applying dissolved fertilizer to crops during their growth stages through an irrigation system (Nasim et al., 2012). Fertigation is the significant component of drip irrigation system and worldwide it has been proved beneficial method for crop fertilization, particularly under arid and semi-arid regions; and more particularly under water stress conditions (Wajid et al., 2017). Integration of fertigation with broadcasting @ $175 \mathrm{~kg} \mathrm{ha}^{-1}$ provided required nitrogen to the crop that is why highest seed yield of sunflower was obtained at this combination of treatments. In the findings of research Rathod et al. (2017) reported that significant respective increase in seed yield of Brassica juncea (L.) was observed by 34.6, 53.1 and $72.1 \%$ under fertigation at 40,80 , and $120 \mathrm{~kg} \mathrm{~N} \mathrm{ha}{ }^{-1}$ over control. Moreover, lower seed yield of sunflower at control (no nitrogen) and other treatments might be due to deficit nitrogen availability to crop plants during the critical growth stages causing retardation of vegetative and reproductive growth and non-utilization of carbohydrates for end product synthesis. Genetic variability is very crucial item for selection of crop variety (Sujatha et al., 2002). Genetic similarities and differences existing in the genotypes are utilized efficiently as genetic resource in the breeding programs (Safavi, 2011). Hybrids of sunflower are more stable and highly self-fertile with a high yield performance and greater uniformity at maturity (Kaya and Atakisi, 2004). In our study genotype HO-1 surpassed Hysun-39 almost in all growth and yield traits, particularly seed yield $\left(\mathrm{kg} \mathrm{ha}^{-1}\right)$. This superiority of HO-1 over Hysun-39 might be attributed to genetic potential and better adoptability of open pollinated variety (HO-1) under agro-ecological conditions of Tandojam. The early sunflower cultivars, having the highest yield can be recommended for the similar ecological conditions (Ozturk et al., 2017). Sunflower maturation under different environmental conditions would accumulate different concentration of oleic acid. Similarly, significant variations in linoleic acid content have also been observed among locations, planting dates and sunflower hybrids by Ahmad and Qureshi (2001). The variability in genetics of crop has much importance in breeding programs (Sujatha et al., 2002). In some cases, the hybrid genotypes have been found to be superior in terms of seed yield to their parental lines (Shahsavari et al., 2010). The highest 1000-seed weight and seed yield ( $\mathrm{kg}$ ha $\left.{ }^{1}\right)$ were recorded from sunflower genotype Nsovak (Kandil et al., 2017).

\section{Conclusions and Recommendations}

The outcomes of present research showed that nitrogen application@ $125 \mathrm{~kg} \mathrm{ha}^{-1}$ in three splits $(1 / 3$ broadcasting at sowing time + fertigation $-1 / 3$ at $1^{\text {st }}$ and $1 / 3$ at $3^{\text {rd }}$ irrigation) demonstrated substantial increase in seed yield $\left(\mathrm{kg} \mathrm{ha}^{-1}\right)$ of sunflower over other treatments. Amongst the sunflower genotypes, more production potential was found in $\mathrm{HO}-1$ which resulted in higher seed yield $\left(\mathrm{kg} \mathrm{ha}^{-1}\right)$ in contrast to Hysun-39.

\section{Acknowledgement}

The research article is the prepared from the original $\mathrm{PhD}$ research work of the $1^{\text {st }}$ author. The study was undertaken at the Sindh Agriculture University Tandojam.

\section{Novelty Statement}

Application of nitrogen to sunflower through fertigation in combination with broadcasting is a unique research under agro-climatic conditions of Tandojam, Sindh, Pakistan. 
Authors' Contribution

Imran Ali Chandio: Conducted the trial, collected data and wrote article.

Muhammad Nawaz Kandhro: Supervised the scholar throughout the experiment and preparation of the article.

Qamaruddin Jogi: Gave his input in write-up of manuscript. G.M. Jamro supplied the research material.

Siraj Ahmed Channa: Contributed in statistical analysis of data.

\section{Conflict of interest}

The authors have declared no conflict of interest.

\section{References}

Ahmad, G.Z. and A. Qureshi. 2001. Response of sunflower hybrid to different planting dates at Peshawar Valley. Sarhad J. Agric., 17: 561-564.

Alves, L.S., M. Elvia, L. Martinez, S.Z. Everaldo, M. Silvestre, F. Andre M.S. Dos and S.D.S. Regina. 2017. Different nitrogen and boron levels influence the grain production and oil content of a sunflower cultivar. Acta Sci. Agron. Maringa, 39(1): 59-66. https://doi. org/10.4025/actasciagron.v39i1.30941

Amin, M., A. Razzaq, R. Ullah and M. Ramzan. 2006. Effect of planting methods, seed density and nitrogen phosphorus fertilizer levels on sweet corn (Zea mays L.). J. Res. Sci., 17 (2): 83-89.

Asad, M.E, S.R, Clemente, A.D. Gupta and R.H.K.G. Loof. 2002. Impacts of fertigation via sprinkler irrigation on nitrate leaching and corn yield in an acid-sulphate soil in Thailand. Agri. Water Manage., 52: 197-213. https://doi. org/10.1016/S0378-3774(01)00136-6

Buriro, M., A.S. Sanjrani, Q.I. Chachar, N.A. Chachar, S.D. Chachar, B. Buriro, A.W. Gandahi andT. Mangan. 2015. Effect of water stress on growth and yield of sunflower. Int. J. Agric. Technol., 2(1): 260- 270.

Daniela, D., C.L. Coelho, A.O.M. Júnior, K.B.D. Silva, N.D.S. Dias and R.O. Batista. 2018. Fertigation of sunflower crops using landfill leachate. Caatinga Mossoro, 31(4): 944- 953. https://doi.org/10.1590/198321252018v31n417rc

Debaeke, P., P. Casadebaig and N.B. Langlade.
2021. New challenges for sunflower ideotyping in changing environments and more ecological cropping systems. Oils Fats Crops Lipids, 28: 1-23. https://doi.org/10.1051/oc1/2021016

GoP. 2020. Pakistan Economic Survey, Ministry of Finance Division, Islamabad 2019-20, Economic Advisory Wing Islamabad Pakistan, pp. 21-22.

GoP. 2019. Pakistan Economic Survey, Ministry of Finance Division, Islamabad 2018-19, Economic Advisory Wing Islamabad Pakistan, pp. 19-20.

Kaya, Y. and I.K. Atakisi. 2004. Combining ability analysis of some yield characters ofsunflower (Helianthus annuus L.). Helia, 27(41):75-84. https://doi.org/10.2298/HEL0441075Y

Keerio, R.A., N.S. Soomro, A.A. Soomro, M.A. Siddiqui, M.T. Khan, G.S. Nizamani, M.N. Kandhro, M. Siddiqui, H. Khan and F.D. Soomro. 2020. Effect of foliar spray of zinc on growth and yield of sunflower (Helianthus annuus L.). Pakistan J. Agric. Res., 33(2): 264-269. https://doi.org/10.17582/journal. pjar/2020/33.2.264.269

Khan, S. and Inamullah. 2019. Tillage instruments and phosphorus levels enhance grain and oil yield in sunflower. Sarhad J. Agric., 35(2): 491-499. https://doi.org/10.17582/journal. sja/2019/35.2.491.499

Kandil, A.A., A.E. Sharief and A.M.A. Odam. 2017. Response of some sunflower hybrids (Helianthus annuus L.) to different nitrogen fertilizer rates and plant densities. Int. J. Environ. Agric. Biotech., 2 (6): 2456-1878. https://doi.org/10.22161/ijeab/2.6.26

Kumar, D.S., R. Sharma and A.S. Brar. 2021. Optimising drip irrigation and fertigation schedules for higher crop and water productivity of oilseed rape (Brassica napus L.). Irrigation Sci., 39: 535-548. https://doi.org/10.1007/ s00271-020-00714-y

Mahmood, A., M.F. Saleem, M. Tahir, M.A. Sarwar, T. Abbas, A. Zohaib and H.T. Abbas. 2018. Sunflower (Helianthus annuus L.) growth, yield and oil quality response to combined application of nitrogen and boron. Pak. J. Agric. Res., 31(1): 86-97. https://doi.org/10.17582/ journal.pjar/2018/31.1.86.97

Masood, M.S. 2014. Oilseed Crops Pakistan Member Plant Sciences PARC May 22, 2014.

Nasim, W., A. Ahmad, H.M. Hammad, H.J. 
Chaudhary and M.F.H. Munis. 2012. Effect of nitrogen on growth and yield of sunflower under semi-arid conditions of Pakistan. Pak. J. Bot., 44(2): 639-648.

Ozturk, E., T. Polat and M. Sezek. 2017. The effect of sowing date and nitrogen fertilizer form on growth, yield and yield components in sunflower. Turkish J. Field Crops, 22(1): 143151. https://doi.org/10.17557/tjfc.312373

PARC, 2019. Sunflower. http://www.parc.gov. pk/index.php/en/csi/137-narc/crop-sciencesinstitue/718-sunflower. Accessed 01 Dec. 2019.

Rathod, S.S., K. Shekhawat, B.K. Kandpal and O.P. Premi. 2017. Improvement of physiological and productivity traits of Indian mustard (Brassica juncea) through micro-irrigation and fertigation under hot semi-arid eco-region. Indian J. Agric. Sci., 87(9):1257-1262.

Rayan, J., G. Estefan and A. Rashid. 2001. Soil and plant analysis laboratory manual. international center for agricultural research in the Dry Areas (ICARDA), Aleppo, Syria. pp. 172.

Safavi, S.A. 2011. Genetic variability of some morphological traits in sunflower (Helianthus annuus L.). Am. J. Sci. Res., 17: 19-24.

Shahsavari,M.R.,M.Nikobin and M.Karimi.2010. Final evaluation of new hybrid of sunflowers. First National Symposium of Oil Grain Plants, Isfahan University of Technology, Isfahan, Iran. 84156-83111.

Shabbir, R.N., M.Y. Ashraf, E.A. Waraich, R. Ahmad and M. Shahbaz. 2015. Combined effects of drought stress and NPK foliar spray on growth, physiological processes and nutrient uptake in wheat. Pak. J. Bot., 4(7): 1207-1216.

Sheoran, P., V. Sardana and R. Singh. 2014. Evaluating productivity potential of spring planted sunflower (Helianthus annuus L.) hybrids in response to sowing time under changing climate scenario. Indian. J. Agron., 59 (1): 124- 132 .

Statistix. 2006. Statistix 8.1 user guide, version 1.0. Analytical Software, PO Box 12185, Tallahassee FL 32317 USA. Copyright 2006 by Analytical Software.

Sujatha, H.L., Chikkadevaiah and Nandini. 2002. Genetic variability study in sunflower inbreds. Helia, 25 (37): 93-100.https://doi.org/10.2298/ HEL0237093S

Ullah, S., A. Muhammad and S.M. Wazir. 2015. Effect of Hydropriming duration on growth and yield of maize landrace in Bannu, KPK, Pakistan. Pak. J. Plant. Sci., 16(2): 99-105.

Wajid, N., A. Ahmad, S. Ahmad, M. Nadeem, N. Masood, M. Shahid, M. Mubeen, G. Hoogenbooma and S. Fahad. 2017. Response of sunflower hybrids to nitrogen application grown under different agro-environments. J. Plant Nutri., 40 (1): 82-92. https://doi.org/10 .1080/01904167.2016.1201492

Yassen, A.A., Abdallah, E.F and M. S. Gaballah. 2011. Response of sunflower plants to nitrogen fertilizers and phytohormones under drainage water irrigation. Aust. J. Basic. Appl. Sci., 5(9): 801-807. 